\title{
Important role of interferon regulatory factor (IRF)-3 in the interferon response of mouse macrophages upon infection by Newcastle disease virus
}

\author{
HOLGER WILDEN ${ }^{1}$, VOLKER SCHIRRMACHER ${ }^{1,2}$ and PHILIPPE FOURNIER ${ }^{1}$ \\ ${ }^{1}$ German Cancer Research Center, Im Neuenheimer Feld 280, 69120 Heidelberg, Germany
}

Received February 8, 2011; Accepted March 30, 2011

DOI: $10.3892 /$ ijo.2011.1033

\begin{abstract}
Newcastle disease virus (NDV) is an interesting agent for activating innate immune activity in macrophages including secretion of TNF- $\alpha$ and IFN- $\alpha$, upregulation of TRAIL and activation of NF- $\mathrm{BB}$ and iNOS. However, the molecular mechanism of such cellular activities remains largely unknown. Tumor selectivity of replication of NDV has been described to be linked to deviations in tumor cells of the type I interferon response. We therefore focused on the interferon response to NDV of macrophages as part of innate anti-viral and anti-tumor activity. In particular, we investigated the functional significance of the interferon regulatory factor genes (IRF)-3 and IRF-7. Deletion of the IRF-3 or IRF-7 gene was found to increase susceptibility of mouse macrophages to virus infection. Surprisingly, NDV replicated better in IRF-3 KO than in IRF-7 KO macrophages. Further analysis showed that IRF-3 KO macrophages have a lower basal and NDV-induced RIG-I expression in comparison to IRF-7 KO macrophages. This might explain why, in IRF-3 KO macrophages, the secretion of type I interferons after NDV infection is delayed, when compared to IRF-7 KO and wild-type macrophages. In addition, IRF-3 KO cells showed reduced NDV-induced levels of IRF-7. This effect could be prevented by priming the cells first by interferon- $\alpha$. Further results indicated that an early production of type I interferon rather than high maximal levels at later time points are important for resistance to infection by NDV. In conclusion, these results demonstrate an important role of IRF-3 for the innate antiviral response to NDV of mouse macrophages.
\end{abstract}

Correspondence to: Professor Volker Schirrmacher, ${ }^{2}$ Present address: Center of Immunology and Oncology (IOZK), Hohenstaufenring 30-32, 50674 Köln, Germany

E-mail: v.schirrmacher@dkfz.de

Dr Philippe Fournier, German Cancer Research Center (DKFZ), Im Neuenheimer Feld 280, 69120 Heidelberg, Germany

E-mail: p.fournier1@yahoo.de

Key words: innate, virus infection and replication, type I interferon, interferon regulatory factor 3 , interferon regulatory factor 7

\section{Introduction}

Macrophages are large mononuclear phagocytic cells present in a resting state in many tissues that participate directly or indirectly in diverse biological functions including tissue remodeling, inflammation, immunity, clearance of apoptotic cells, healing and angiogenesis. They also play a dual role in host defense by triggering the innate immune response and contributing to the development of the adaptive immune response through cytokine secretion and immune cell stimulation. When properly activated, macrophages can exert tumoricidal activities that are mediated through phagocytosis, antibodydependent cell cytotoxicity and production of interleukin (IL)-1, tumor necrosis factor (TNF), TNF-related apoptosisinducing ligand (TRAIL), FasL, nitric oxide (NO) and oxygen radicals (reviewed in ref. 1). Because the activation of these cells represents a major host defense mechanism against tumor cells which is independent of tumor antigen recognition, the broad anti-tumor activation of macrophages by Newcastle disease virus (NDV) is of major interest for active non-specific immunotherapy in cancer patients.

NDV is an avian paramyxovirus that preferentially replicates in cancer cells and that is being applied in cancer patients since decades (reviewed in ref. 2). A defective interferon response, which is observed in many neoplastic cells, gives tumor cells a selective growth advantage by making them less sensitive to growth-inhibitory effects of type 1 interferon [IFN; especially the main 2 forms: the interferon- $\alpha$ (IFN- $\alpha$ ) and $-\beta$ (IFN- $\beta)$ ] in their microenvironment. It has been shown that defects in the interferon response make neoplastic cells susceptible to infection by NDV (3). In contrast, in normal cells, a strong interferon response is induced and blocks viral replication (3). We reported previously (4) on the capacity of NDV to activate anti-tumor activity in murine macrophages in vitro (chromium release assays or $\left[{ }^{3} \mathrm{H}\right]$-thymidine release tests) using a broad range of tumor cells as the target. Upon transfer to tumor-bearing mice, such properly activated macrophages caused anti-metastatic effects (4). The underlying mechanism(s) of the activation of macrophages remains largely unknown although NO production has been linked to $\mathrm{NF}-\kappa \mathrm{B}$ activation (5).

Originally recognized for their capacity to protect against viral infection, it is now clear that IFN- $\alpha / \beta$ are important 
immunomodulators, linking the innate and adaptive arms of the immune response $(7,8)$. In addition to their role in pathogeninduced diseases, type I IFN plays a role in preventing the development and the progression of cancer (6). Their diverse biological effects as well as their cytotoxic and antiproliferative properties have made type I IFNs very attractive candidates for anticancer therapy. Recombinant forms of interferons have been largely exploited in particular as adjuvants in combination with cancer vaccines for the in vivo differentiation or activation of immune cells. Once properly activated, macrophages kill tumor cells and help to generate an effective tumor-targeted immune response. Passive immunotherapy by re-infusion into the patient of ex vivo generated and highly active monocytederived macrophages may help in the re-establishment of an anti-tumor state $(7,8)$. Macrophages activated with IFN- $\gamma$ have been proven feasible and safe in phase I and phase II clinical trials for the treatment of several malignancies including mesothelioma, ovarian and bladder cancer (9-11). This appears as an attractive complement to conventional cancer therapies. Macrophages can also play an important role in the subversion of the immune system by the tumor by developing into M2 type macrophages (12). In this respect, NDV appears as an ideal agent for the prevention of M2 type macrophages activation since it has been shown to favor the induction of M1 type macrophages, expressing for instance TRAIL.

In the present study, we analyzed the functional significance of some key genes of the interferon response for NDV susceptibility and antiviral gene expression in murine macrophages. Among them, interferon regulatory factor (IRF)-3 is constitutively expressed and not further upregulated upon viral infection (13). One other important factor, IRF-7, is constitutively expressed only in plasmacytoid dendritic cells (PDC) $(14,15)$ but is induced upon viral infection in most other cell types. In the signaling pathway triggered by cytosolic RNA-helicases [retinoic acid inducible gene I (RIG-I) and melanoma differentiation antigen 5 (MDA-5)], IRF-7 is activated in addition to IRF-3. The role played by IRF-7 and IRF-3 in cells upon viral infection was exhaustively analyzed and appears important. However, a detailed analysis of the interferon response of macrophages upon NDV infection remains to be addressed. To this end, we studied the importance of both proteins, IRF-3 and IRF-7, for the replication of NDV in macrophages and for the induction of type I interferons. The expression of these 2 proteins appears to be associated with the resistance of normal cells to NDV infection. We analyzed in vitro the response of macrophages from mice which had been knocked out of the IRF-3 and IRF-7 genes.

This study describes important details of the mechanism of interferon induction in macrophages upon NDV infection. It highlights the crucial role played by the transcription factor IRF-3 and suggests an alternative approach to increase macrophage activation and anti-tumor functions via the manipulation of the expression of this protein.

\section{Materials and methods}

Mice, generation of macrophages and cell lines. Wild-type C57BL/6 mice were purchased from Charles River Laboratories (Sulzfeld, Germany). All transgenic mice strain had a C57BL/6 background. The IRF-3, IRF-7 knock-out and the IRF-3 IRF-7 double knock-out mice were kindly provided by Dr A. Krug (Institute for Medical Microbiology, Immunology and Hygiene, Technical University Munich, Germany). The

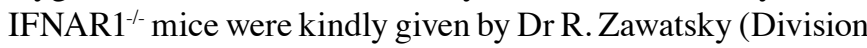
of Viral Transformation Mechanisms, DKFZ, Heidelberg, Germany).

Murine macrophages were generated by growing bone marrow cells flushed from femora. The freshly harvested cells were then seeded $\left(4 \times 10^{6}\right.$ cells per petri-dish having a $10-\mathrm{cm}$ diameter) cell culture and were grown for 8-12 days in RPMI1640 medium supplemented with $10 \%$ FCS and $20 \% \mathrm{v} / \mathrm{v}$ M-CSF containing supernatant from L929 cells. After 10 days, the adherent cells were recovered from the cell culture plates and the macrophage phenotype determined by flow cytometry after staining with a monoclonal anti-F4/80 antibody (Cedarlane Laboratories, Burlington, Canada). Expression of the F4/80 macrophage marker was found in $>80-90 \%$ of the cells. To generate the L929 supernatant, a dense L929 cell monolayer was split 1:10 and then grown for one week in DMEM with $10 \%$ FCS. Then the supernatant was harvested, centrifuged ( $250 \mathrm{x} \mathrm{g}, 5 \mathrm{~min})$ and used in further experiments. Macrophages were also derived following the same procedures from BALB/c and DBA/2 mice (obtained from Charles River Laboratories). Lung tissue and liver tissue were also tested. The tumor cell lines: CT26p, CT26wt, J774A.1 (here called J774), RAW 264.7 (here called RAW), DA3, ESb and B16 (all obtained from the DKFZ tumor bank) were kept in culture in RPMI with 5\% FCS and glutamine, penicillin and streptomycin as additives.

Viruses and infections. Lentogenic NDV Ulster 2C (named NDV Ulster in this study) was obtained in 1984 from Dr P. H. Russel [Department of Microbiology and Parasitology, Royal Veterinary College, University of London (England)]. Recombinant NDV expressing the jellyfish-enhanced green fluorescent protein (EGFP), named NDFL-EGFP, was generated from a cDNA clone of the lentogenic NDV strain LaSota as described (16) and was kindly provided by Dr B. Peeters (Division of Infectious Diseases, Animal Sciences Group, Wageningen UR, AB Lelystad, The Netherlands). Both NDV viruses were propagated in embryonated chicken eggs, harvested from the allantoic fluid, purified by ultra-centrifugation and characterized as described (17). Quantification was performed by hemagglutination (HA) assay. The smallest virus concentration leading to visible agglutination of sheep erythrocytes is defined as 1 hemagglutination unit (HU). For infection, cell suspensions were washed twice with FCS-free medium and $1 \times 10^{6}$ cells were incubated with $10 \mathrm{HU}$ of NDV in a final volume of $100 \mu \mathrm{l}$ for $1 \mathrm{~h}$ at $37^{\circ} \mathrm{C}$ in a cell culture incubator. During the incubation, cells were shaken every $15 \mathrm{~min}$. The cells were then washed twice and cultured further in $2 \mathrm{ml}$ medium with $10 \%$ FCS in a 12 -well plate. To generate UV-inactivated NDV, the virus suspension was exposed to UV light for $5 \mathrm{~min}\left(254 \mathrm{~nm}, 2 \mathrm{~mW} / \mathrm{cm}^{2}, 7 \mathrm{~cm}\right.$ distance).

Antibodies, IFN- $\alpha$, sera and flow cytometry. Monoclonal anti-F4/80-PE antibodies were obtained from Cedarlane Labs. Mouse rIFN- $\alpha 4$ was obtained from the culture medium of Chinese hamster ovary (CHO) cells transfected with an 
expression vector encoding mouse IFN- $\alpha 4$ cDNA and was provided by Dr Rainer Zawatzky (DKFZ). For analysis by flow cytometry, $4 \times 10^{5}$ cells per sample were used. The fluorescence was detected by using a FACScan flow cytometer (BectonDickinson, Heidelberg, Germany). All antibodies were diluted in FACS buffer (PBS containing 5\% FCS and $0.1 \% \mathrm{NaN}_{3}$ ). Cells were stained by incubation with the mAbs for $20 \mathrm{~min}$ on ice in the dark. Cells were washed twice with FACS buffer between each incubation with an antibody. All FACS data were analyzed with the WinMDI 2.8 software (Joe Trotter, The Scripps Research Institute, CA, USA).

RNA isolation, cDNA preparation and real-time PCR. Cell pellets were either frozen at $-80^{\circ} \mathrm{C}$ after removal of the medium or used immediately for RNA preparation. Cells were disrupted using a rotor-stator homogeniser. Total RNA was extracted using the RNeasy mini kit (Qiagen, Hilden, Germany) according to the manufacturer's instructions. Total RNA (500 ng) was then reversely transcribed into cDNA using oligo(dT)12-18 primers and the SuperScript ${ }^{\mathrm{TM}}$ II reverse transcriptase (both Invitrogen, Karlsruhe, Germany) according to the manufacturer's instructions. For each assay, $500 \mathrm{ng}$ total RNA per sample were reversely transcribed using oligo(dT)12-18 primers and the generated cDNA was used in subsequent PCR reactions. For the real-time PCR, primers which bound just once in the genome when tested in a database search (BLAST, NCBI) were designed. The primers were optimised to show a melting curve with one peak only and to have approximately the same amplification efficiency as the gene used for normalisation. The master mix was prepared as recommended by the manufacturer (Eurogentec, Seraing, Belgium) and the concentrations for the primers and for $\mathrm{MgCl}_{2}$ were adjusted. Real-time RT-PCR was carried out using a Gene Amp ${ }^{\circledR} 5700$ Sequence Detection System (Applied Biosystems, Weiterstadt, Germany). After an initial incubation of $25-\mu 1$ reaction mix (Eurogentec) for $10 \mathrm{~min}$ at $95^{\circ} \mathrm{C}$, amplification was performed for 45 cycles consisting of a 15 -sec hold at $95^{\circ} \mathrm{C}$ and a $1-\mathrm{min}$ hold at $60^{\circ} \mathrm{C}$. The expression of the target genes was normalised with the respective housekeeping gene after it had been ensured that the amplification efficiency was the same. For the viral $M$ gene and $\beta$-actin detection, a fluorescent oligonucleotide probe system was used, while the other genes were detected with SYBR-Green. The sequences of the primers and the probes were previously described (3).

IFN- $\alpha$ and IFN- $\beta$ enzyme-linked immunosorbent assay (ELISA) and encephalomyocarditis virus (EMCV) protection assays. IFN- $\alpha$ in supernatants of cultured cells was determined with ELISA using a rat monoclonal antibody and a rabbit polyclonal antibody against murine IFN- $\alpha$ (PBL Interferon Source, NJ, USA). IFN- $\beta$ was measured by ELISA using a rat monoclonal antibody specific for murine IFN- $\beta$ (United States Biological, MA, USA) and a rabbit polyclonal antibody against murine IFN- $\beta$ (PBL Interferon Source). For the determination of both proteins, peroxidase-conjugated donkey anti-rabbit IgG $(\mathrm{H}+\mathrm{L}) \mathrm{F}\left(\mathrm{ab}^{\prime}\right) 2$ fragment (Jackson ImmunoResearch Laboratories, PA, USA) was added to the well before adding the TMB substrat. The encephalomyocarditis virus (EMCV) protection assays were performed as described (3) using EMCV virus and L929 indicator cells. The dilution of a super- natant that protected $50 \%$ of the cells against virus-induced lysis contained by definition one international unit (IU) IFN.

Statistical analysis. All analyses were performed with the GraphPad Prism 4.0 software (GraphPad Software, San Diego, CA, USA). P-values were calculated with a two-sided Student's t-test.

\section{Results}

Association between NDV replication and IRF-3 and IRF-7 expression in normal and neoplastically transformed macrophages. We first tested the susceptibility to infection by NDV of bone marrow-derived primary macrophages and two macrophage-derived tumor cell lines J774 and RAW 264. The cells were infected either with the lentogenic strain NDFLEGFP or with the mesogenic strain NDFLtag-EGFP. These are recombinant NDVs with a marker gene, the enhanced green fluorescent protein (EGFP) inserted in the viral genome. Transcription of viral genes thus leads to expression of green fluorescent protein which can be used as indicator of viral replication. One day after infection, the EGFP signal of the cells was measured by flow cytometry. Fig. 1A shows that the RAW tumor cells had the highest EGFP signal while normal macrophages had the lowest signal. Analysis by real-time RT-PCR of the viral $M$ gene expression after infection confirmed the results obtained with the EGFP signal (data not shown). Fig. 1B shows that IRF-3 was expressed significantly higher in normal cells than in tumor cells before infection (basal) and after infection with NDV (induced). The average basal IRF-3 expression (part a) was 64 relative expression units (REU) for normal and 7 REU for tumor cells. Real-time RT-PCR analysis revealed that the mean NDV-inducible IRF-3 expression (part b) was 32 REU for normal and 4 REU for tumor cells. The basal and NDV-inducible IRF-3 expression turned out to be significantly higher in normal than in tumor cells. Subsequently the IRF-7 gene expression was investigated. Fig. 1Bc and demonstrate that the basal and NDV-induced IRF-7 expression was also significantly higher in normal than in tumor cells. The average basal IRF-7 expression (c) was $83 \mathrm{REU}$ in normal and $11 \mathrm{REU}$ in tumor cells, and the average inducible IRF-7 expression (d) 5841 REU for normal and 240 REU for tumor cells.

Importance of IRF-3 and IRF-7 for resistance to NDV infection. In order to assess the importance of key molecules of the interferon response for resistance against NDV infection, primary macrophages were generated from different $\mathrm{KO}$ mice. The genes which were absent were the ones coding for the transcription factors IRF-3 or IRF-7. Macrophages deleted of the gene coding for the type I interferon receptor (IFNAR) were used as control. The different cultures of primary bonemarrow-derived macrophages were infected with NDV (strain NDFL-EGFP). Viral matrix (M) gene and EGFP transgene expression was determined $24 \mathrm{~h}$ later by real-time RT-PCR (Fig. 2Aa) and flow cytometry (Fig. 2Ab), respectively. Viral $\mathrm{M}$ gene expression was low in the wild-type macrophages and EGFP expression was seen in $<3 \%$ of these cells. In contrast, $>40 \%$ of the IFNAR KO macrophages expressed EGFP. The NDV M level in these macrophages was 2000 times higher 
A

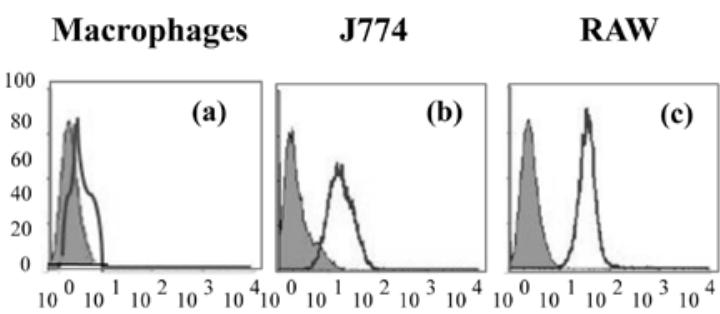

(\% of

the max)

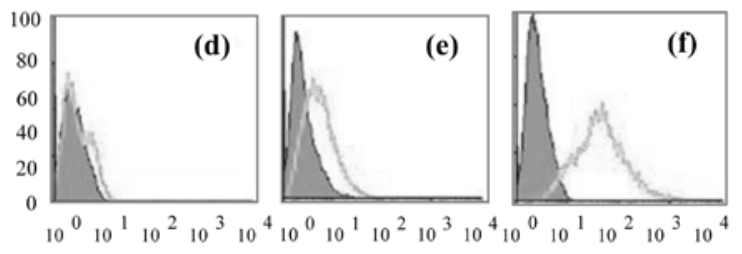

B

FL (AFU)

(a) Basal

IRF3

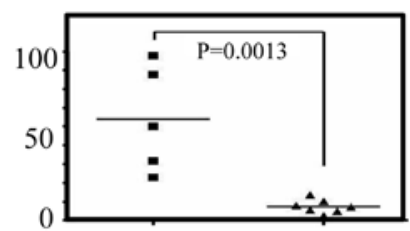

(c)

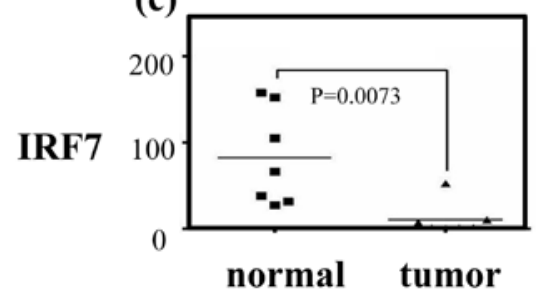

(b)

Induced

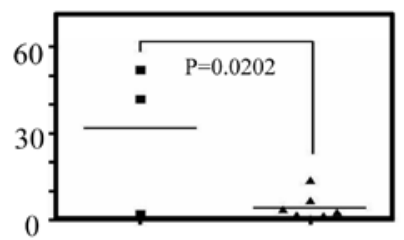

(d)

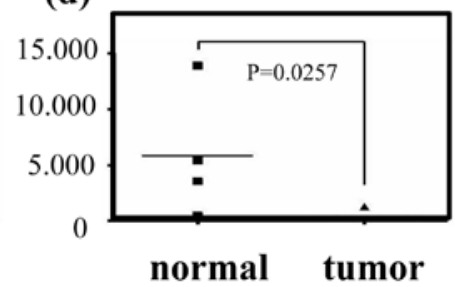

Figure 1. Replication of NDV in different murin macrophages-derived tumor cell lines and in primary cells and analysis of the basal and inducible IRF-3 expression in primary and tumor cells before and after NDV infection. (A) Tumor selective replication of NDV in macrophages-linked tumor cell lines. Bone marrow-derived macrophages from BALB/c mice, J774A.1 and RAW 264.7 tumor cells were infected with 10 HU per 10 ${ }^{6}$ cells NDFL-EGFP (top) or NDFLtag-EGFP (bottom). Twenty-four hours after infection with the recombinant EGFP-expressing NDV LaSota, EGFP expression was measured by flow cytometry. Shown is one representative experiment out of three. (B) Analysis of the basal and inducible IRF-3 expression in primary and tumor cells before and after NDV infection. The IRF-3 and IRF-7 expression level was measured in several primary and tumor cell types before and $24 \mathrm{~h}$ after infection with 10 HU NDV Ulster or NDFL-EGFP. The gene expression was determined by real-time RT-PCR with the comparative CT method using the acidic ribosomal phosphoprotein PO for normalisation. The basal (a) and induced (b) IRF-3 expression of several primary [for (a) macrophages from Balb/c, C57BL/6 and DBA/2 mice, lung tissue and liver tissue from C57BL6, for (b): spleen, macrophages from Balb/c and C57BL/6 mice] and tumor cell types [for (a) and (b) CT26p, CT26wt, J774A.1, RAW 264.7, DA3, Esb and B16] is shown. In a similar way, the basal (c) and induced (d) IRF-7 expression of several primary [for (c) and (d) spleen cells, macrophages from BALB/c, C57BL/6 and DBA/2 mice] and tumor cell type [for (c) and (d): CT26wt, J774A.1, RAW 264.7, DA3, Esb and B16] is shown. The p-value was calculated using an unpaired Student's t-test. Bars indicate standard deviation. The correlation coefficient was calculated with the mean expression values.

than in the wild-type macrophages. NDV M gene expression was highly increased also in IRF-3 and IRF-7 KO macrophages. It was $\sim 5$ times higher in the IRF-3 than in the IRF-7 KO macrophages. In order to analyze the kinetics of NDV infection in the IRF-3 and IRF-7 gene KO macrophages, viral M gene expression was determined at different time points after infection with NDV (Fig. 2B). In addition to the single $\mathrm{KO}$ macrophages, IRF-3 and IRF-7 double KO macrophages were included in the NDV infection kinetics experiments. NDV $\mathrm{M}$ gene expression observed at 2 and $4 \mathrm{~h}$ after infection with NDV Ulster was low in all the different KO cells tested (Fig. 2B). Eigth hours post-infection, viral $\mathrm{M}$ gene expression in the wild-type and IRF-7 KO macrophages increased by a factor of 10. Thereafter, it decreased to a low but stable level. In contrast, the macrophages derived from the IRF-3 IRF-7 double KO (DKO) mice and from the IFNAR KO mice showed during the first $18 \mathrm{~h}$ of infection a 10.000 -fold increase in $\mathrm{M}$ gene expression. NDV M gene expression in the IRF-3 KO macrophages increased up to $8 \mathrm{~h}$ after infection to a level that was approximately intermediate between the one observed for the wild-type and the IFNAR KO macrophages.

Influence of IRF-3 and IRF-7 on the constitutive and NDVinduced RIG-I expression. We then evaluated the expression of RIG-I, the main cytoplasmic receptor for recognition of foreign viral RNA, with real-time RT-PCR. Compared to wildtype macrophages, we observed a significantly lower RIG-I expression in IRF-3, IRF-3/7 and IFNAR KO macrophages 
A
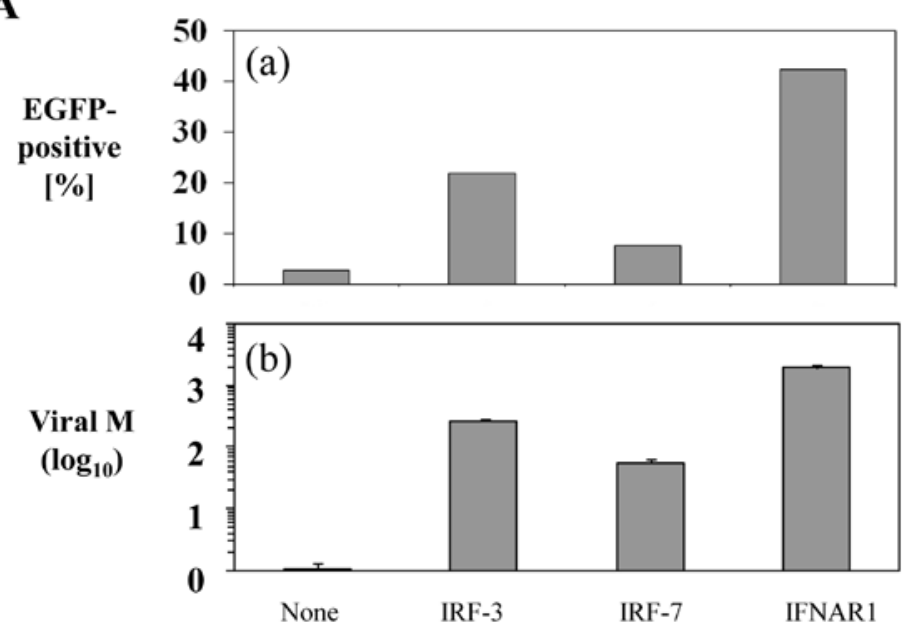

Gene knock-out

B

Gene(s) knock-

out:

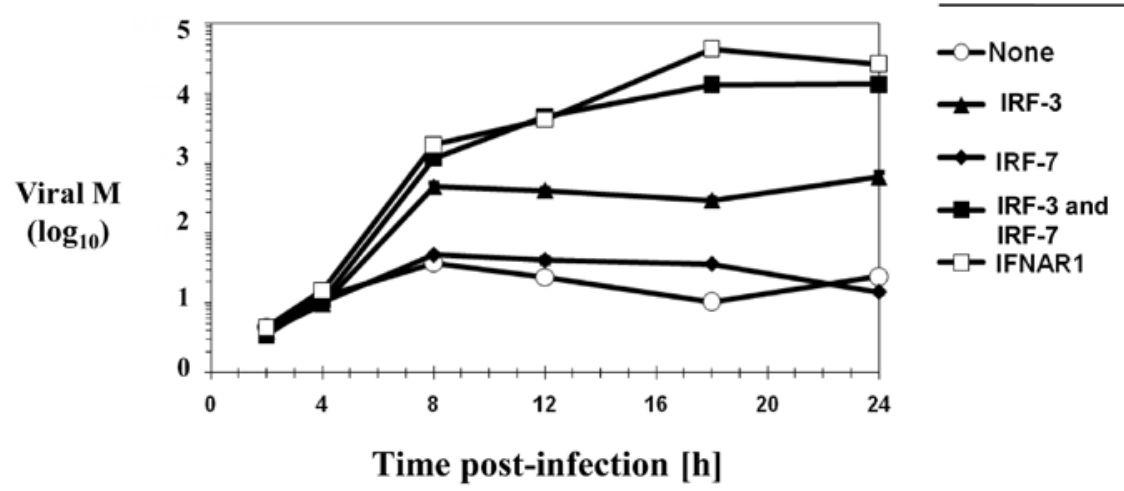

Figure 2. Replication of NDV in macrophages generated from IRF-3 and IRF-7 gene knock-out mice. Wild-type macrophages and macrophages with knockouts for different interferon-related genes were infected with NDV. (Aa) Twenty-four hours after infection with the recombinant EGFP-expressing NDV LaSota, (a) EGFP expression was measured by flow cytometry. Shown is one representative experiment out of three. (b) At the same time point, NDV M gene expression was determined with real-time RT-PCR using the harvested cell pellets. The results are represented as the mean of three measurements. Bars indicate standard deviation. (B) NDV M gene expression was determined with real-time RT-PCR at different time points after infection with NDV Ulster. The results are presented as the mean of three measurements. Bars indicate standard deviation.

(Fig. 3A). In contrast, RIG-I expression was not significantly reduced in the IRF-7 KO macrophages. Thus, it appears that the constitutive RIG-I expression in the KO macrophages correlates inversely with their susceptibility to infection by NDV. The kinetics of RIG-I expression after infection by NDV revealed a delay of RIG-I expression in the IRF-3 KO cells compared to the wild-type and also to the IRF-7 KO cells (Fig. 3B). This delay was particularly apparent $4 \mathrm{~h}$ postinfection. The highest RIG-I expression in the IRF-3 KO cells was lower than in the wild-type or in the IRF-7 KO cells. In the IRF-3/7 and IFNAR KO macrophages, RIG-I expression remained low until $12 \mathrm{~h}$ post-infection. After this time point RIG-I expression was strongly increased in the IRF-3/7 KO and slightly increased in the IFNAR KO macrophages. Hence, the expression kinetics of RIG-I correlated inversely with susceptibility to infection by NDV in the wild-type and in the KO macrophages. Next, we investigated the induction of RIG-I expression in the KO macrophages $8 \mathrm{~h}$ after co-culture with UV-inactivated NDV (UV-NDV). UV inactivation abro- gates the ability of NDV to replicate (data not shown). UV-NDV clearly induced RIG-I expression in the wild-type and in all KO macrophages except for the IRF-3/7 and IFNAR KO cells (Fig. 3C). RIG-I induction was strongest in the wild-type cells $8 \mathrm{~h}$ post-infection. In comparison to wild-type macrophages the RIG-I expression level $8 \mathrm{~h}$ after NDV infection was lower in the $\mathrm{KO}$ cells with $45 \%$ in IRF-3 KO, 31\% in IRF-7 KO and $12 \%$ in IFNAR KO cells. Eighteen hours after infection the increase was generally lower than $8 \mathrm{~h}$ after NDV infection. RIG-I expression at this time point was slightly higher in the IRF-3 and IRF-7 KO than in the wild-type macrophages. In summary, IRF-3 appeared to be more important than IRF-7 for a high constitutive RIG-I expression level and an early (4 h) RIG-I response after infection by NDV. The reduction of RIG-I expression in the IRF-3/7 double KO cells was generally higher than the sum of the reduction in the IRF-3 and IRF-7 KO cells. Both transcription factors are therefore important for the virus-induced response of up-regulating the level of viral RNA recognition receptor. 
A

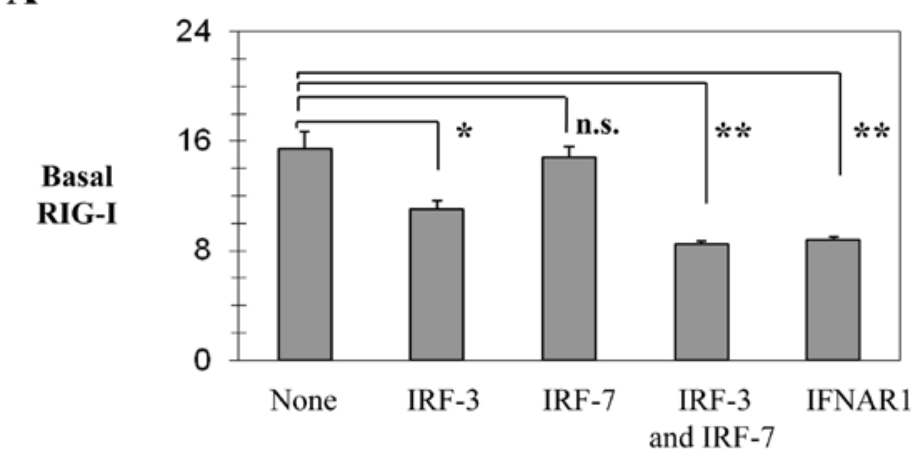

B

Gene(s) knock out

Gene(s)

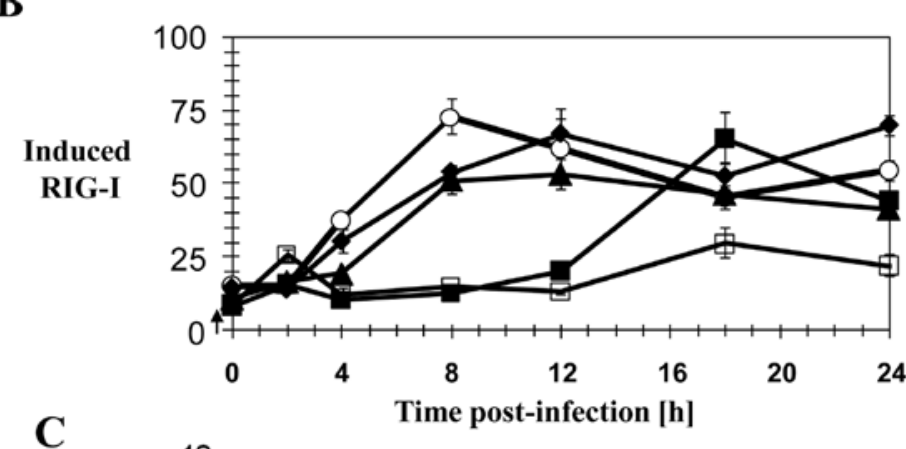

knock-out:

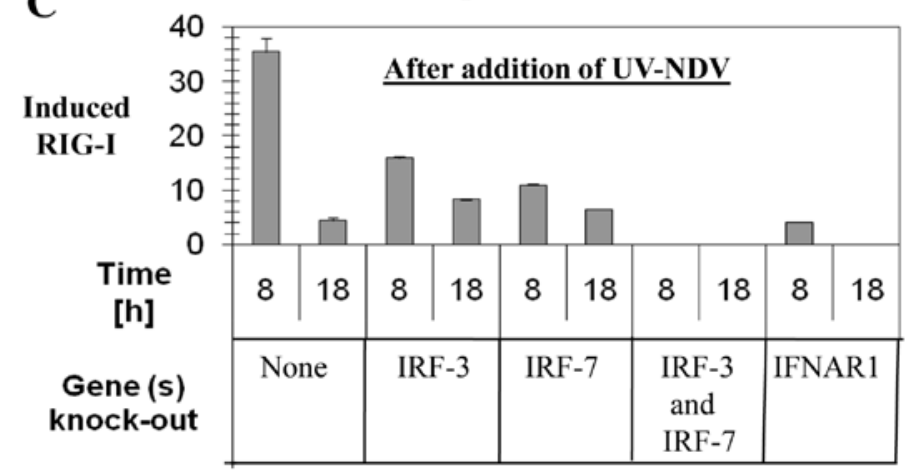

$\infty$ None

\pm IRF-3

$\rightarrow$ IRF-7
$\rightarrow$ IRF-3
- and

IRF-7

七 IFNAR1

Figure 3. Constitutive and NDV-induced expression of RIG-I in the knock-out macrophages. RIG-I expression was assessed in the different knock-out macrophages with real-time RT-PCR. (A) Basal RIG-I expression in the absence of NDV infection. P-values were calculated in relation to the basal RIG expression in the wild-type. ${ }^{*} \mathrm{p}<0.01,{ }^{* *} \mathrm{p}<0.001$; NS, not significant ( $\mathrm{p}=0.51$ ). (B) RIG-I expression at different time points after infection with NDV Ulster. (C) Expression of RIG-I after 8 and $18 \mathrm{~h}$ of co-culture with UV-inactivated NDV Ulster. The results are presented as the mean of three measurements. Bars indicate standard deviation.

Role of IRF-3 and IRF-7 in the secretion of type I interferon after infection by $N D V$. The secretion of type I interferon upon NDV infection is crucial for a successful antiviral response via the induction of antiviral molecules or of the transcription factor IRF-7. The generation of type I interferon in wild-type and $\mathrm{KO}$ macrophages following infection by NDV was determined with different types of assays. The amount of virus neutralizing type I interferon in the supernatants of infected cells was determined with the EMCV protection assay. It was found that in the IRF-3 KO macrophages the secretion of type I interferon was delayed (Fig. 4A). At early time points (up to $8 \mathrm{~h}$ after infection by NDV), the type I interferon level was lower than in the wild-type cells. However, at $18 \mathrm{~h}$ postinfection, the secreted type I interferon level was significantly higher in the IRF-3 KO cells than in the wild-type. With respect to the IRF-7 KO cells, the secreted type I interferon level was always lower than that of the wild-type or that of the
IRF-3 KO macrophages. IRF-3/7 double KO and the IFNAR $\mathrm{KO}$ cells produced almost no type I interferon.

Next, we determined via ELISA the supernatant content of IFN- $\alpha$ (Fig. 4B) and IFN- $\beta$ (Fig. 4D). IFN- $\alpha$ could be detected only in the supernatants of wild-type and IRF-3 KO cells (Fig. 4B). IFN- $\alpha$ secretion reached 2.4 times higher maximal levels in the IRF-3 KO cells than in the wild-type cells. No IFN- $\alpha$ was found in the IRF-7, IRF-3/7 and IFNAR KO. Realtime RT-PCR analysis of the IFN- $\beta$ mRNA expression after infection by NDV showed a similar picture as the ELISA assays (Fig. 3C). The expression of IFN- $\beta$ in the IRF-3 KO cells was delayed up to $8 \mathrm{~h}$ after infection. It increased to a maximal level that was $\sim 2.4$ times higher than that in the wild-type macrophages (Fig. 4C) before the mRNA level decreased again. As shown in Fig. 4D, there was a delayed secretion of IFN- $\beta$ in the IRF- $3 \mathrm{KO}$ cells. Up to $8 \mathrm{~h}$ there was almost no IFN- $\beta$ produced by the IRF-3 KO macrophages. But, from 

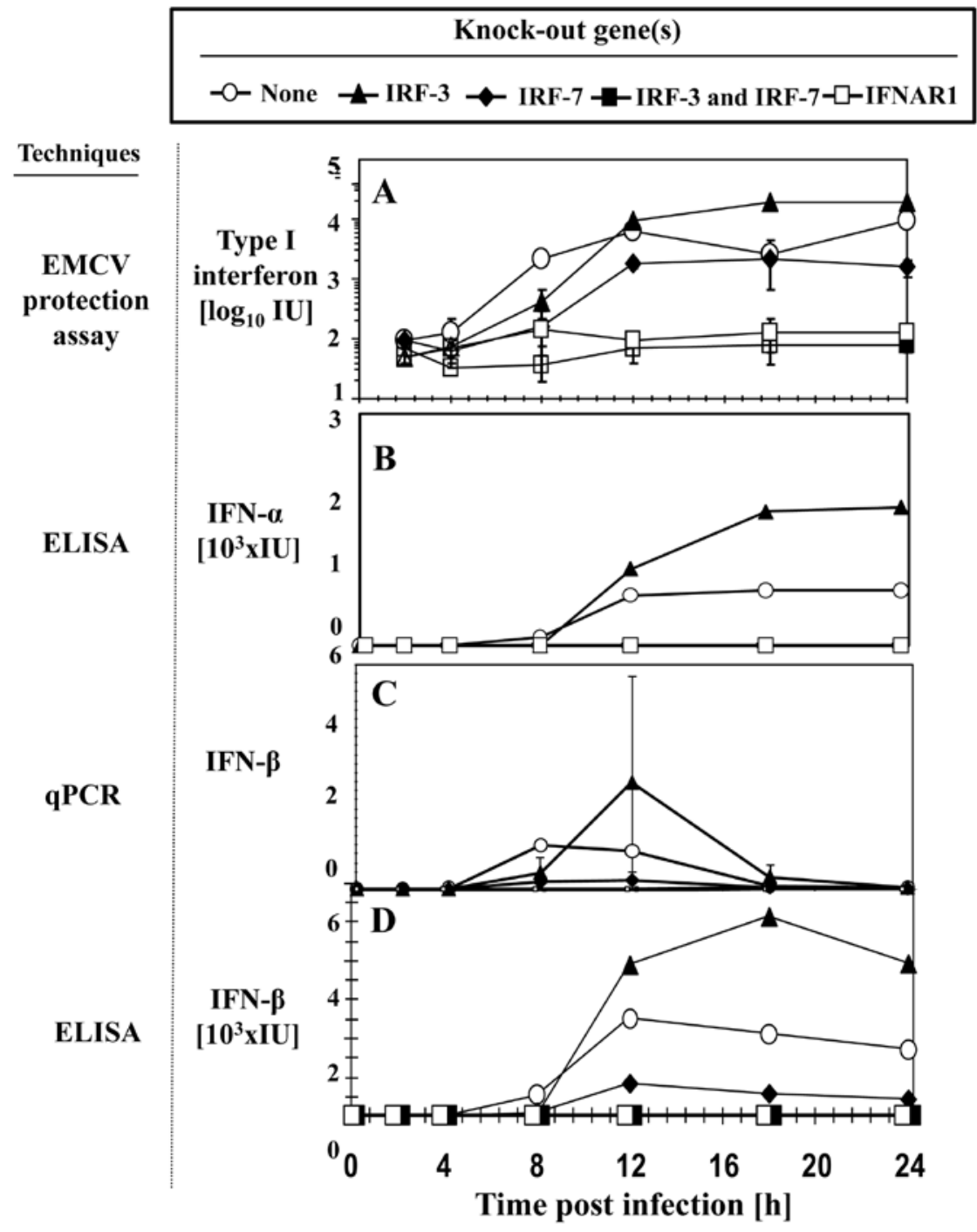

Figure 4. Kinetics of the interferon secretion in the knock-out macrophages after infection with NDV. (A) The amount of type I interferons was assessed with an EMCV protection assay in the supernatant of the knock-out macrophages at different time points after infection with NDV Ulster. (B and D) The amount of IFN- $\beta$ and IFN- $\alpha$ in the supernatants was measured with ELISA. (C) Gene expression of IFN- $\beta$ after NDV infection was determined with real-time RT-PCR. The results are presented as the mean of two (A) or three (C) measurements, respectively. Bars indicate standard deviation.

$12 \mathrm{~h}$ on the IFN- $\beta$ level was higher in IRF-3 KO than in the wild-type macrophages. The maximal IFN- $\beta$ level in the IRF-3 KO macrophages (51500 IU) was about twice as high as that in the wild-type macrophages (25200 IU). In the IRF-7 $\mathrm{KO}$ cells, the maximal IFN- $\beta$ level was intermediate $(8300$ IU). Virtually no IFN- $\beta$ could be found in the supernatants from the IRF-3/7 and in the IFNAR KO cells. The IRF-7 cells differed markedly from the IRF3 cells with regard to their IFN- $\beta$ response. There was only a slight increase in IFN- $\beta$ expression with a maximum that was $\sim 20 \%$ that of the wildtype. Maximum IFN- $\beta$ expression appeared between 8 and $12 \mathrm{~h}$ after infection and decreased subsequently to almost basal levels at $24 \mathrm{~h}$. Almost no IFN- $\beta$ could be detected with the IRF-3/7 and IFNAR KO cells. Comparison of Fig. 4C and $\mathrm{D}$ reveals a difference in the kinetics of expression levels of IFN- $\beta$ mRNA (C) and protein (D). The latter seems much more stable. The IFN- $\beta$ levels determined by ELISA (Fig. 4D) were, however, higher than the virus neutralizing interferon levels depicted in Fig. 4A. While the EMCV protection assay measures only bioactive interferon, the ELISA depends only on the binding of interferon to the used antibody. So the ELISA might also measure partly degraded interferons that have lost their bioactivity.

The results suggest that an early production of type I interferon rather than high maximal interferon levels at later time points are important for resistance to infection by NDV. When interferon generation was delayed, as in the IRF-3 KO cells, virus replication apparently progressed too far to be effectively inhibited at later time points after infection, even with type I interferon levels that were substantially higher than in the wild-type. In contrast to the induction of RIG-I expression, a co-culture with UV-NDV did not lead to the generation of type I interferon, neither in the wild-type nor in any of the KO macrophages (data not shown).

Influence of IRF-3 on the NDV-induced expression of IRF-7. IRF-7 mRNA expression was also followed by real-time RT-PCR. A strong upregulation was observed in wild-type 
and IRF-3 KO macrophages after infection by NDV (Fig. 5A). The maximal IRF-7 level was very similar in both macrophage types and was reached $24 \mathrm{~h}$ after NDV infection. Yet, the kinetics of increase in the IRF-7 expression were different. The response was slower in the IRF-3 KO than in the wildtype macrophages. Between 4 and $18 \mathrm{~h}$ after infection, IRF-7 expression in the IRF-3 KO cells was about half compared to the wild-type cells. Thus, IRF-3 appeared to be crucial for the timely induction of IRF-7. The IRF-7 response to NDV infection was strongly reduced in IFNAR KO macrophages. There was, however, a slight increase in IRF-7 expression, pointing towards interferon-independent mechanisms of IRF-7 mRNA up-regulation. Upon co-culture with UV-NDV, there was also an induction of IRF-7 mRNA expression in wild-type cells (Fig. 5B). In the IRF-3 KO macrophages, however, the induction by UV-NDV of IRF-7 expression was substantially lower and reached only $\sim 25 \%$ of the maximum in the wild-type. No induction was seen in IFNAR KO cells. Compared to infection by live NDV, the induction of IRF-7 expression during co-culture with UV-NDV was $\sim 8$ times lower in the wild-type and around 30 times lower in the IRF-3 KO macrophages. This suggests that the amplification of viral danger signals by NDV replication was responsible for $88-97 \%$ of the induced IRF-7 expression. The low expression of IRF-7 in the IRF-3 KO cells points towards the importance of IRF-3 for the UV-NDV-induced expression of IRF-7.

Influence of IFN- $\alpha$ on NDV infection and expression of RIG-I and IRF-7 in IRF-3 knock-out macrophages. An effective interferon response to virus infection depends on positive feedback mechanisms mediated by interferons via IFNAR. Secreted interferons act in an auto- and paracrine manner to activate these feedback loops. In order to test the influence of IRF-3 and the responsiveness to interferons, IRF-3 KO macrophages were pre-treated with IFN- $\alpha$ before infection with NDV.

To estimate viral replication first, NDV M gene expression was tested $24 \mathrm{~h}$ after infection of macrophages pre-treated or not with IFN- $\alpha$ (Fig. 6A). It was found that the IFN- $\alpha$ pre-treatment significantly reduced $\mathrm{M}$ gene expression in the wild-type and in all $\mathrm{KO}$ macrophages except for the IFNAR $\mathrm{KO}$ cells. $\mathrm{M}$ gene expression was reduced to about the same level in wild-type, IRF-3 and IRF-7 KO cells. In IRF-3/7 double $\mathrm{KO}$ cells, the absolute $\mathrm{M}$ gene expression was $\sim 20$ times higher than in wild-type cells. IFN- $\alpha$ pre-treatment of such cells also led to a reduction of M gene expression. In IFNAR $\mathrm{KO}$ cells, the absolute $\mathrm{M}$ gene expression was similarly high as in IRF-3/7 KO cells but pre-treatment with IFN- $\alpha$ could not affect $\mathrm{M}$ gene expression in these cells. Pre-treatment with IFN- $\alpha$ strongly increased RIG-I mRNA expression $8 \mathrm{~h}$ after infection by NDV. This was true for the wild-type macrophages and for the IRF-3 and IRF-7 KO cells (Fig. 6B). With respect to the IRF-3/7 double KO cells, the level of constitutive and IFN- $\alpha$-induced RIG-I expression was much lower. The IFNAR KO cells responded only marginally to IFN- $\alpha$ treatment. Pre-treatment with IFN- $\alpha$ also induced an increase in IRF-7 mRNA in wild-type and IRF-3 KO macrophages (Fig. 6B). This increase was clearly stronger in the IRF-3 KO than in the wild-type cells. This could indicate a regulatory mechanism of IRF-7 expression involving IRF-3. No induction of IRF-7 by IFN- $\alpha$ was seen in IFNAR KO macrophages.

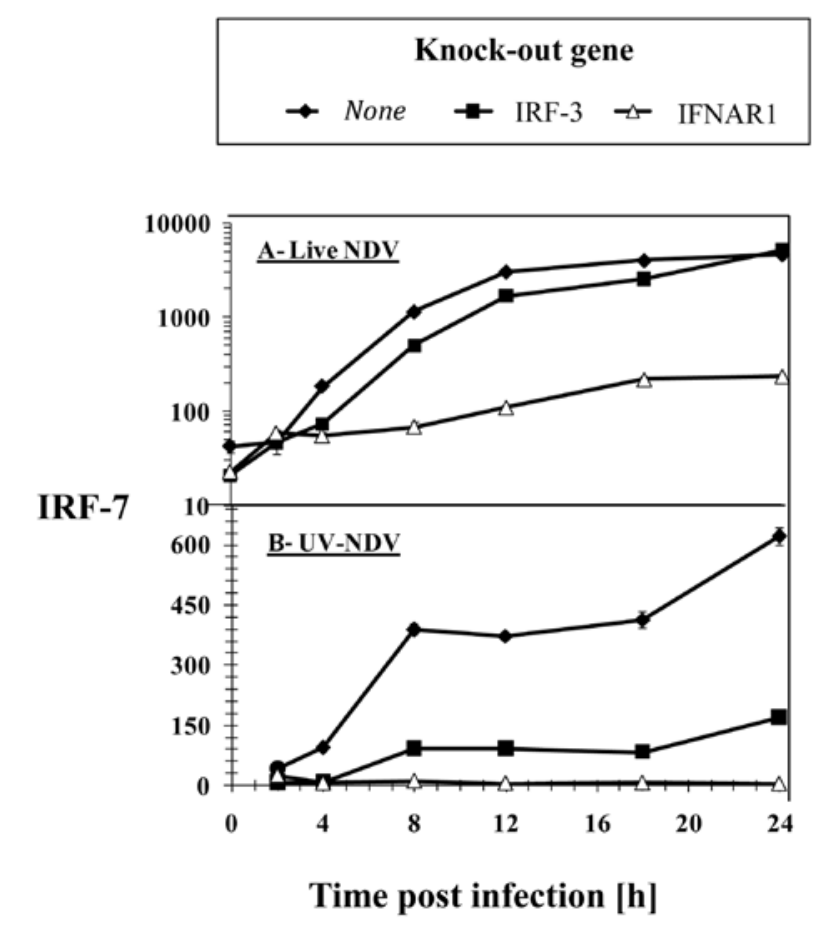

Figure 5. Expression of IRF-7 in primary macrophages derived from different knock-out mice. (A) Expression of IRF-7 was determined by realtime RT-PCR at different time points after infection with NDV Ulster. (B) Expression of IRF-7 at different time points after co-culture with UV-inactivated NDV Ulster. The results are presented as the mean of three measurements. Bars indicate standard deviation.

\section{Discussion}

In the present study, we demonstrated a lower expression of both IRF-3 and IRF-7 in macrophage-derived tumor cells versus normal macrophages. This difference makes the tumor cells less resistant to infection by NDV. We also analyzed the effects of deleting genes coding for these transcription factors on the replication behaviour of NDV in mouse macrophages. Deletion of IRF-3 and IRF-7 in macrophages led to a strong increase in the production of viral $\mathrm{M}$ gene transcripts. Interestingly, these two important transcription factors for the induction of type I interferons appeared to be important at distinct time points following virus infection. The absence of IRF-3-limited antiviral gene expression in the early but not in the late phase of the interferon response, while IRF-7 appeared to be crucial for the strong expression of antiviral genes in the late phase after virus infection. These functional analyses revealed a crucial importance of constitutive IRF-3 levels and early interferon responsiveness for resistance to NDV infection.

The key for the induction of a cellular interferon response against the danger that represents infection by NDV is its recognition. The two interferon regulatory factors we analyzed in this study, IRF-3 and IRF-7, are the two most important transcription factors for the induction of type I interferons. They are activated by cytosolic RNA helicases such as RIG-I as well as by TLRs upon viral infection. In normal macrophages, IRF-3 expression was relatively high and increased slightly after NDV infection while, in macrophage-like tumor cells, IRF-3 expression was low and remained low upon infection. 
A

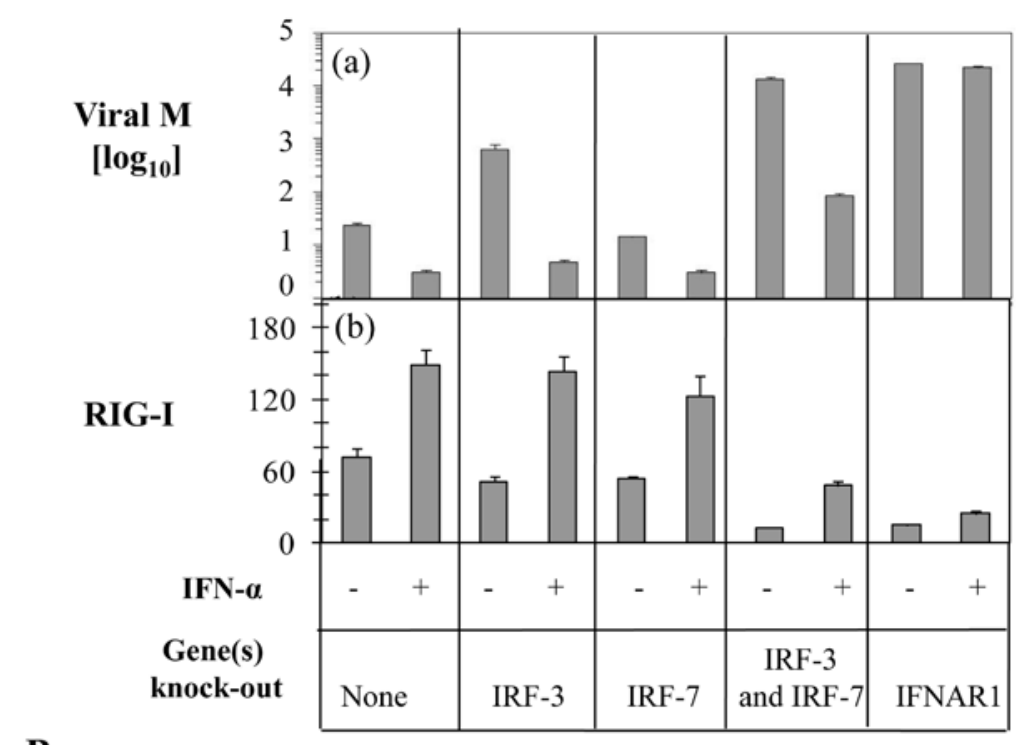

B

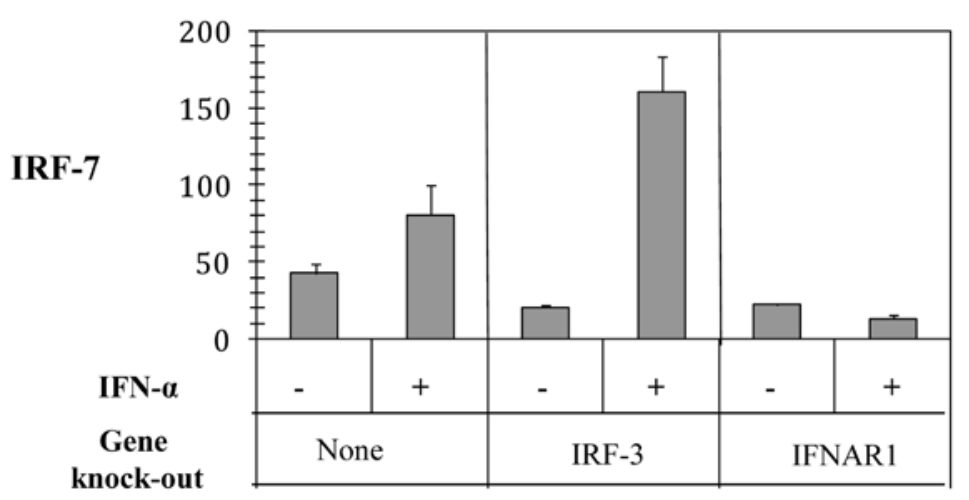

Figure 6. Influence of IFN- $\alpha$ on NDV infection and the expression of RIG-I and IRF-7. Macrophages were cultivated either in normal medium or for $16 \mathrm{~h}$ before infection in medium containing $40 \mathrm{IU} / \mathrm{ml}$ IFN- $\alpha 4$. (A) NDV M gene expression was assessed $24 \mathrm{~h}$ after infection with real-time RT-PCR. (B) RIG-I expression was measured $8 \mathrm{~h}$ after NDV infection. (C) IRF-7 expression was measured immediately after the pretreatment with IFN- $\alpha 4$. The results are presented as the mean of three measurements. Bars indicate standard deviation.

These results are in accordance with the general assumption that IRF-3 action is mostly constitutive, as shown for example by Juang et al (19). In the different macrophage cells analyzed, there was a correlation between NDV resistance and IRF-3 expression, suggesting a role of this molecule for NDV resistance of cells (3). A low expression of IRF-3 in tumor cells could lead to a diminished signal transduction after the detection of viral danger signals and would lead to a weaker antiviral response. This hypothesis was corroborated by the analysis of several normal and tumor cells types. IRF-3 expression in normal cells was significantly higher at the basal as well as at the inducible level than in tumor cells and there was a strong negative correlation between IRF-3 expression and NDV susceptibility (3).

In contrast to IRF-3, IRF-7 was found to be highly inducible by NDV infection in macrophages, corroborating findings described elsewhere $(20,21)$. In the macrophage cell types, we observed a correlation between NDV resistance and IRF-7 expression level after NDV infection, indicating IRF-7 as a further factor involved in NDV resistance (3). This conclusion was corroborated by the analysis of several normal and tumor cell types. IRF-7 was significantly higher expressed in normal cells and, in tumor cells, there was a strong negative correlation between IRF-7 expression and NDV susceptibility. It is a crucial molecule for the late phase of the antiviral responses. Its importance for the enhancement and diversification of IFN induction has been confirmed in earlier studies (22). They showed a correlation between IRF-7-deficiency and a severely impaired type I IFN response in systemic HSV-1 and EMCV infections. They also demonstrated that although IRF-3 participates in the MyD88-independent type I IFN induction pathway, its contribution is minor in the absence of IRF-7.

It has even been proposed that IRF-7 is the master regulator of type I interferon-dependent immune responses and that all elements of IFN responses, whether the systemic production of IFN in innate immunity or the local action of IFN from pDCs in adaptive immunity, are under the control of IRF-7 (18). IRF-7 is often targeted by viruses to limit antiviral responses and to increase virus survival and replication. For example, a Kaposi's sarcoma-associated herpes viral protein inhibits virus-mediated induction of type I interferon by blocking IRF-7 phosphorylation and nuclear accumulation (23). Also 
the herpes simplex virus ICP0 RING finger domain inhibits IRF-3- and IRF-7-mediated activation of interferon-stimulated genes (24). Often viral proteins increase the degradation of IRF-7 such as the rotavirus NSP1 protein (25) or the KSHV immediate-early transcription factor RTA that encodes a ubiquitin E3 ligase activity that targets IRF-7 for proteasomemediated degradation (26).

When macrophages were treated with IFN- $\alpha 4$, we observed in this study that there was a strong induction of IRF-7 expression, whereas the IRF-3 expression did not change. This is in accordance with the view that IRF-3 is constitutively expressed while IRF-7 is highly inducible. The expression of IRF-7 is thought to be induced by early IFNs and depends on the ISRE present in the IRF-7 promoter (27). In addition IFN-independent mechanisms for the induction of IRF-7 by virus infection have been suggested (28). In this study, we observed that the increase in expression of IRF-7 induced by IFN- $\alpha 4$ correlated with NDV resistance and was much higher in normal macrophages than in the $\mathrm{IFNAR}^{-/-}$macrophages. These results showed that exogenous IFN has a strong effect on virus infection. This phenomenon was partially inhibited when both IRF-3 and IRF-7 were deleted and totally blocked when the INFAR was knocked out.

The functional importance of IRF-3 and IRF-7 for NDV resistance and the interferon response became apparent in the analysis of knock-out macrophages. The absence of both transcription factors increased the susceptibility of macrophages to infection by NDV. The increase of viral $\mathrm{M}$ gene expression was higher in the IRF-3 than in the IRF-7 knock-out cells. Virus replication was comparable at early time points. Thereafter it increased in the IRF-3 knock-out cells while it decreased in the IRF-7 knock-out cells. This suggests that the secretion of early IFNs, which depends on IRF-3, is more important for limiting NDV infection than the late secretion of high amounts of all IFN types. Furthermore the lack of IRF-3 clearly delays and limits the expression of IRF-7 so that until later time points the IRF-3 knock-out macrophages display almost an IRF-3/7 double knock-out phenotype and are therefore especially vulnerable to NDV infection. On the other hand IRF-7 knock-out macrophages express almost the same amount of IRF-3 as the wild-type and are therefore not inhibited in their ability to establish the early phase of the interferon response. The IFN- $\alpha 4$ pre-treatment was sufficient to limit NDV replication in the IRF-3 and IRF-7 knock-out macrophages almost as strongly as in the wild-type macrophages. It seems that, in the tested experimental setting, no additional secretion of IFN- $\alpha 4$ or of other type I IFNs mediated by IRF-3/7 was necessary to strongly inhibit NDV replication. However, it cannot be concluded that IFN- $\alpha 4$ is the only IFN that plays a role in the interferon response to NDV infection. It is known that NDV infection stimulates the expression of several IFN- $\alpha$ subtypes (20). Unfortunately, a real-time RT-PCR assay could not be established to elucidate the expression of different IFN- $\alpha$ genes at different time points after NDV infection. There are more than a dozen IFN- $\alpha$ genes in the mouse and it proved to be very difficult to find primer systems that detect only one member of this gene family.

The IFN secretion upon NDV infection was clearly reduced in IRF-7 knock-out macrophages, while IRF-3 knock-out macrophages showed an IFN secretion similar to the wild- type macrophages. This underlines the role of IRF-7 as the master regulator of the IFN response but confirms also that for NDV resistance in vitro an early interferon response is more important than the ability of a cell to secrete large amounts of type I IFNs. The IFN- $\alpha 4$ pre-treatment reduced the type I interferon secretion in the IRF-3/7 knock-outs as well as in the wild-type probably because of the reduction of NDV replication and hence the decreased concentration of IFN-stimulating viral danger signals. IRF-3 and IRF-7 did not seem to be required for the induction of RIG-I by NDV infection or by IFN- $\alpha 4$ since the RIG-I expression in the knock-out cells was comparable with the wild-type. Nevertheless, the expression of IFN- $\beta$ was strongly compromised in the IRF-3/7 knock-out cells. In the absence of IRF-3 the IFN- $\beta$ expression was clearly delayed, while almost no IFN- $\beta$ was expressed at any time point after NDV infection when IRF-7 was lacking. This indicates that although IFN- $\beta$ is regarded as an early IFN it requires not only the presence of IRF-3 but also that of IRF-7 for its expression. An initial model suggested that only IRF-3 is involved in the early phase of IFN- $\beta$ induction by forming a multi-protein complex called enhanceosome (29). Meanwhile it could be shown in knock-out mice that the presence of IRF-7 is also crucial for the IFN- $\beta$ induction after HSV, VSV and EMCV infection (18). So the low amounts of IRF-7 that are expected to be present in the absence of virus infection seem to be important for IFN- $\beta$ induction. The high IFN- $\beta$ expression in the IRF-3 KO cells at the latest time point occurred probably because of the higher NDV replication in these cells that could be translated into a stronger response when finally enough IRF-7 had been synthesized.

Analysis of the response after simultaneous deletion of the IRF-3 and IRF-7 gene showed a complementarity between both genes in detecting viral infection and inducing an interferon response. $\mathrm{M}$ gene expression in the IRF-3/7 double $\mathrm{KO}$ cells was much higher than the sum of $M$ gene expression in the IRF-3 and IRF-7 KO macrophages. The cytosolic viral RNA receptor RIG-I is known to be crucial for the induction of an interferon response after infection of cells by NDV. Differences in RIG-I expression could therefore be a reason for differences in the susceptibility to NDV of the different gene KO macrophages. Very recently, the interaction of RIG-I and ZAPS, a member of the poly(ADP-ribose) polymerase family, has been shown to allow the enhancement of the strength and duration of innate antiviral response (30). A difference of levels of ZAPS expression between normal and tumor cells, in addition to the different expression levels of IRF-3 and IRF-7 observed in this study, might be speculated.

The result of this study showing that IRF-3 might act in place of IRF-7 to induce a type I IFN response is challenging, given that it has been shown that IRF-7 can function as a master regulator of type I IFN-mediated responses (18). The IRF-7dependent type I IFN response has been observed not to be essential for early resistance against murine $\mathrm{CMV}$ infection (31). An essential role of IRF-3 for type I IFN induction by HSV-1, vesicular stomatitis virus (VSV) and encephalomyocarditis virus (EMCV) was observed in fibroblasts but not in DC $(18,21)$.

Previous investigations showed that gene delivery of wildtype (WT) IRF-3 or IRF-7 proteins can be sufficient for the 
expression of some IRF target genes; however, only marginal levels of type I IFN are detected in the absence of virus infection (20,32). Romieu-Mourez et al (33) showed that the anti-tumor properties of primary macrophages are stimulated by ectopic expression of a constitutively active form of IRF-7, delivered using a regulated adenovirus vector system. Expression of active IRF-3 5D by primary human macrophages resulted in rapid cell death, whereas expression of IRF-7 247-467 was not as pro-apoptotic and induced long-lasting up-regulation of type I IFN genes. In addition, they observed that macrophages transduced with IRF-7 247-467 displayed enhanced tumor inhibitory effects that were mediated in part by induction and secretion of type I IFNs.

In conclusion, we highlighted in this study the importance of IRF-3 during the induction phase of an interferon response in mouse macrophage upon NDV infection. This may open new strategies of optimization of macrophage activation against tumors. Deletion of IRF-3 via RNA interference can be expected to allow a stronger NDV replication in macrophages leading to their stronger activation against tumor cells. We postulate that inhibition of IRF-3 with subsequent stronger type I IFN production will potentiate the anti-tumor functions of NDV infected macrophages.

\section{Acknowledgements}

We thank Dr Ben Peeters from the Division of Infectious Diseases, Animal Sciences Group, for the recombinant EGFP-expressing NDV. We also acknowledge the kindness of Professor Rainer Zawatzky for providing IFNAR ${ }^{-/-}$mice, for helpful discussions and for critically reading the manuscript. We thank also Dr Anne Krug from the Department of Internal Medicine II, Klinikum Rechts der Isar, Technical University Munich, for providing us the IRF-3, IRF-7 and IRF-3 and IRF-7 knocked out mice. We are grateful to Annette Arnold for her excellent technical assistance. This work was supported partially by the Monsi foundation, Monaco. We gratefully acknowledge also support by the IOZK (www.iozk.de) (Cologne, Germany).

\section{References}

1. Klimp AH, de Vries EG, Scherphof GL and Daemen T: A potential role of macrophage activation in the treatment of cancer. Crit Rev Oncol Hematol 44: 143-161, 2002.

2. Schirrmacher V and Fournier P: Newcastle disease virus: a promising vector for viral therapy, immune therapy, and gene therapy of cancer. Methods Mol Biol 542: 565-605, 2009.

3. Wilden H, Fournier P, Zawatzky R and Schirrmacher V: Expression of RIG-I, IRF-3, IFN- $\beta$ and IRF-7 determines resistance or susceptibility of cells to infection by Newcastle disease virus. Int J Oncol 34: 971-982, 2009.

4. Schirrmacher V, Bai L, Umansky V, Yu L, Xing Y and Qian Z: Newcastle disease virus activates macrophages for anti-tumor activity. Int J Oncol 16: 363-373, 2000.

5. Umansky V, Shatrov VA, Lehmann V and Schirrmacher V: Induction of NO synthesis in macrophages by Newcastle disease virus is associated with activation of nuclear factor-kappa B. Int Immunol 8: 491-498, 1996.

6. Dunn GP, Koebel CM and Schreiber RD: Interferons, immunity and cancer immunoediting. Nat Rev Immunol 6: 836-848, 2006.

7. Ferrantini M, Capone I and Belardelli F: Interferon-alpha and cancer: mechanisms of action and new perspectives of clinical use. Biochimie 89: 884-893, 2007.

8. Moschos S, Varanasi S and Kirkwood JM: Interferons in the treatment of solid tumors. Cancer Treat Res 126: 207-241, 2005.
9. Pagès F, Lebel-Binay S, Vieillefond A, Deneux L, Cambillau M, Soubrane O, Debré B, Tardy D, Lemonne JL, Abastado JP, Fridman WH and Thiounn N: Local immunostimulation induced by intravesical administration of autologous interferon-gammaactivated macrophages in patients with superficial bladder cancer. Clin Exp Immunol 127: 303-309, 2002.

10. Thiounn N, Pages F, Mejean A, Descotes JL, Fridman WH and Romet-Lemonne JL: Adoptive immunotherapy for superficial bladder cancer with autologous macrophage activated killer cells. J Urol 168: 2373-2376, 2002.

11. Monnet I, Breau JL, Moro D, Lena H, Eymard JC, Ménard O, Vuillez JP, Chokri M, Romet-Lemonne JL and Lopez M: Intrapleural infusion of activated macrophages and gammainterferon in malignant pleural mesothelioma: a phase II study. Chest 121: 1921-1927, 2002.

12. Sica A, Larghi P, Mancino A, Rubino L, Porta C, Totaro MG, Rimoldi M, Biswas SK, Allavena P and Mantovani A: Macrophage polarization in tumour progression. Semin Cancer Biol 18: 349-355, 2008

13. Au WC, Moore PA, Lowther W, Juang YT and Pitha PM: Identification of a member of the interferon regulatory factor family that binds to the interferon-stimulated response element and activates expression of interferon-induced genes. Proc Natl Acad Sci USA 92: 11657-11661, 1995.

14. Izaguirre A, Barnes BJ, Amrute S, Yeow WS, Megjugorac N, Dai J, Feng D, Chung E, Pitha PM and Fitzgerald-Bocarsly P: Comparative analysis of IRF and IFN-alpha expression in human plasmacytoid and monocyte-derived dendritic cells. J Leukoc Biol 74: 1125-1138, 2003.

15. Kerkmann M, Rothenfusser S, Hornung V, Towarowski A, Wagner M, Sarris A, Giese T, Endres S and Hartmann G: Activation with CpG-A and CpG-B oligonucleotides reveals two distinct regulatory pathways of type I IFN synthesis in human plasmacytoid dendritic cells. J Immunol 170: 4465-4474, 2003.

16. Al-Garib SO, Gielkens AL, Gruys E, Peeters BP and Koch G: Tissue tropism in the chicken embryo of non-virulent and virulent Newcastle diseases strains that express green fluorescence protein. Avian Pathol 32: 591-596, 2003.

17. Schirrmacher V, Haas C, Bonifer R, Ahlert T, Gerhards R and Ertel C: Human tumor cell modification by virus infection: an efficient and safe way to produce cancer vaccine with pleiotropic immune stimulatory properties when using Newcastle disease virus. Gene Ther 6: 63-73, 1999.

18. Honda K, Yanai H, Negishi H, Asagiri M, Sato M, Mizutani T, Shimada N, Ohba Y, Takaoka A, Yoshida N and Taniguchi T: IRF-7 is the master regulator of type-I interferon-dependent immune responses. Nature 434: 772-777, 2005.

19. Juang YT, Lowther W, Kellum M, Au WC, Lin R, Hiscott J and Pitha PM: Primary activation of interferon A and interferon B gene transcription by interferon regulatory factor 3. Proc Natl Acad Sci USA 95: 9837-9842, 1998.

20. Marie I, Durbin JE and Levy DE: Differential viral induction of distinct interferon- genes by positive feedback through interferon regulatory factor-7. EMBO J 17: 6660-6669, 1998.

21. Sato M, Hata N, Asagiri M, Nakaya T, Taniguchi T and Tanaka N: Positive feedback regulation of type I IFN genes by the IFNinducible transcription factor IRF-7. FEBS Lett 441: 106-110, 1998.

22. Levy DE, Marie I, Smith E and Prakash A: Enhancement and diversification of IFN induction by IRF-7-mediated positive feedback. J Interferon Cytokine Res 22: 87-93, 2002.

23. Zhu FX, King SM, Smith EJ, Levy DE and Yuan Y: A Kaposi's sarcoma-associated herpesviral protein inhibits virus-mediated induction of type I interferon by blocking IRF-7 phosphorylation and nuclear accumulation. Proc Natl Acad Sci USA 99: 5573-5578, 2002.

24. Lin R, Noyce RS, Collins SE, Everett RD and Mossman KL: The herpes simplex virus ICP0 RING finger domain inhibits IRF-3- and IRF-7-mediated activation of interferon-stimulated genes. J Virol 78: 1675-1684, 2004.

25. Barro M and Patton JT: Rotavirus NSP1 inhibits expression of type I interferon by antagonizing the function of interferon regulatory factors IRF-3, IRF5 and IRF-7. J Virol 81: 4473-4481, 2007.

26. Yu Y, Wang SE and Hayward GS: The KSHV immediate-early transcription factor RTA encodes ubiquitin E3 ligase activity that targets IRF-7 for proteosome-mediated degradation. Immunity 22: 59-70, 2005.

27. Lu R, Au WC, Yeow WS, Hageman N and Pitha PM: Regulation of the promoter activity of interferon regulatory factor-7 gene. Activation by interferon and silencing by hypermethylation. J Biol Chem 275: 31805-31812, 2000. 
28. Ning S, Huye LE and Pagano JS: Regulation of the transcriptional activity of the IRF-7 promoter by a pathway independent of interferon signaling. J Biol Chem 280: 12262-12270, 2005.

29. Maniatis T, Falvo JV, Kim TH, Kim TK, Lin CH, Parekh BS and Wathelet MG: Structure and function of the interferon-beta enhanceosome. Cold Spring Harb Symp Quant Biol 63: 609-620, 1998.

30. Hayakawa S, Shiratori S, Yamato H, Kameyama T, Kitatsuji C, Kashigi F, Goto S, Kameoka S, Fujikura D, Yamada T, Mizutani T, Kazumata M, Sato M, Tanaka J, Asaka M, Ohba Y, Miyazaki T, Imamura M and Takaoka A: ZAPS is a potent stimulator of signaling mediated by the RNA helicase RIG-I during antiviral responses. Nat Immunol 12: 37-44, 2011.
31. Steinberg C, Eisenächer K, Gross O, Reindl W, Schmitz F, Ruland J and Krug A: The IFN regulatory factor 7-dependent type I IFN response is not essential for early resistance against murine cytomegalovirus infection. Eur J Immunol 39: 1007-1018, 2009.

32. Barnes BJ, Richards J, Mancl M, Hanash S, Beretta L and Pitha PM: Global and distinct targets of IRF 5 and IRF-7 during innate response to viral infection. J Biol Chem 279: 45194-45207, 2004.

33. Romieu-Mourez R, Solis M, Nardin A, Goubau D, BaronBodo V, Lin R, Massie B, Salcedo M and Hiscott J: Distinct roles for IFN regulatory factor (IRF)-3 and IRF-7 in the activation of antitumor properties of human macrophages. Cancer Res 66: 10576-10585, 2006. 